Cahiers $d u$ MONDE RUSSE

\section{Cahiers du monde russe}

Russie - Empire russe - Union soviétique et États indépendants

49/2-3 | 2008

Sortie de guerre

\title{
La genèse des transferts de populations dans les territoires libérés
}

L'évacuation « volontaire » des Ukrainiens de Pologne vers l'URSS (septembre 1944-juin 1945)

\section{Catherine Gousseff}

\section{(2) OpenEdition}

12 Journals

Édition électronique

URL : https://journals.openedition.org/monderusse/9140

DOI : 10.4000/monderusse. 9140

ISSN : $1777-5388$

Éditeur

Éditions de l'EHESS

\section{Édition imprimée}

Date de publication : 20 septembre 2008

Pagination : 515-536

ISBN : 978-2-7132-2196-5

ISSN : $1252-6576$

Référence électronique

Catherine Gousseff, «La genèse des transferts de populations dans les territoires libérés », Cahiers du monde russe [En ligne], 49/2-3 | 2008, mis en ligne le 01 janvier 2011, consulté le 02 septembre 2022. URL : http://journals.openedition.org/monderusse/9140 ; DOI : https://doi.org/10.4000/ monderusse. 9140 
chercher : repérer : avancer

Cet article est disponible en ligne à l'adresse :

http://www.cairn.info/article.php?ID REVUE=CMR\&ID NUMPUBLIE=CMR 49\&ID ARTICLE=CMR 4920515

La genèse des transferts de populations dans les territoires libérés. L'évacuation « volontaire » des Ukrainiens de Pologne vers l'URSS

par Catherine GOUSSEFF

\section{Editions de l'EHESS | Cahiers du monde russe}

2008/2-3 - Vol 49

ISSN 1252-6576 | ISBN 9782713221965 | pages 515 à 536

Pour citer cet article :

-Gousseff C., La genèse des transferts de populations dans les territoires libérés. L'évacuation « volontaire 》 des Ukrainiens de Pologne vers l'URSS (septembre 1944-juin 1945), Cahiers du monde russe 2008/2-3, Vol 49, p. 515-536.

Distribution électronique Cairn pour les Editions de l'EHESS.

(C) Editions de l'EHESS. Tous droits réservés pour tous pays.

La reproduction ou représentation de cet article, notamment par photocopie, n'est autorisée que dans les limites des conditions générales d'utilisation du site ou, le cas échéant, des conditions générales de la licence souscrite par votre établissement. Toute autre reproduction ou représentation, en tout ou partie, sous quelque forme et de quelque manière que ce soit, est interdite sauf accord préalable et écrit de l'éditeur, en dehors des cas prévus par la législation en vigueur en France. Il est précisé que son stockage dans une base de données est également interdit. 


\section{LA GENÈSE DES TRANSFERTS DE POPULATIONS DANS LES TERRITOIRES LIBÉRÉS}

\section{L'évacuation « volontaire » des Ukrainiens de Pologne vers l'URSS (septembre 1944-juin 1945)}

La libération, par l'Armée rouge, des régions frontalières polono-soviétiques à l'été 1944, a confirmé de facto l'annexion des anciens territoires orientaux de la Pologne, entreprise entre 1939 et 1941 dans le cadre du pacte germano-soviétique. L'établissement de la nouvelle frontière orientale de la Pologne le long de la rivière Bug, à plus de deux cents kilomètres à l'ouest de la ligne tracée au début des années 1920, s'est concrétisé, dès septembre 1944, par la mise en œuvre de vastes transferts de populations ethniques. Le « marqueur » frontalier allait ainsi commencer à être forgé par le déplacement des hommes avant d'être fixé par un traité bilatéral puis par le balisage policier du territoire.

Cette «chirurgie démographique » visait, d'une part, les minorités polonaises installées dans les trois républiques ayant hérité, chacune, d'une portion des anciens territoires polonais, soit la Lituanie, la Biélorussie et l'Ukraine et, d'autre part, les minorités lituaniennes, biélorusses et ukrainiennes de Pologne, encouragées à rejoindre leur république tutélaire soviétique. Dans les faits, cependant, les transferts de populations furent très inégalement réalisés. En effet, en raison des nombreuses contraintes administratives mises à leur départ, seule une part des minorités polonaises quittèrent ou purent quitter la Lituanie et la Biélorussie. De même que de Pologne, les minorités biélorusse et plus encore lituanienne ne revinrent que très symboliquement dans leur patrie respective. A contrario, les transferts de populations furent très intenses de part et d'autre de la nouvelle frontière polono-ukrainienne. En l'espace de près de deux ans, de septembre 1944 à l'été 1946, plus de 800000 Polonais quittèrent l'Ukraine et près de 500000 Ukrainiens de Pologne prirent la route de l'est. Un véritable échange de populations fut ainsi effectué à cette frontière. Il signalait, certes, l'importance des minorités concentrées de part et d'autre de la nouvelle 
ligne, mais la détermination avec laquelle il fut conduit se rapportait davantage à l'acuité prise par les conflits interethniques dans ces régions. Depuis les grands massacres des Polonais de Volhynie par leurs voisins ukrainiens, en 1943, jusqu'aux attaques sauvages des villages ukrainiens de Pologne, une escalade de violences s'était déployée conduisant à une quasi-guerre civile dans la guerre ${ }^{1}$. L'importance prise par ces conflits révélait la force des mouvements de résistance nationale, qu'ils soient ukrainiens (OUN, UPA) ou polonais (AK), lesquels, tout en s'opposant, ne menèrent pas moins une lutte commune contre la nouvelle présence soviétique ${ }^{2}$. Les deux versants de cette histoire, qu'il s'agisse du départ des Polonais d'Ukraine ou de celui des Ukrainiens de Pologne ont fait chacun l'objet de diverses investigations mais c'est sur le cas de la minorité ukrainienne que les travaux historiographiques sont les plus nombreux et débattus, en particulier au sein des milieux scientifiques polonais ${ }^{3}$. La vivacité du sujet tient, pour beaucoup, à la responsabilité de l'État polonais dans la dispersion violente de cette minorité qui s'effectua en deux temps distincts. D'abord par l'intervention de l'armée polonaise qui, dès septembre 1945 , fut déployée dans les régions orientales du pays et qui, jusqu'à la fermeture de la frontière polono-soviétique à l'été 1946, mena différentes opérations d'expulsions massives des foyers ukrainiens récalcitrants vers l'Ukraine soviétique. Malgré la radicalité de ces actions, au moins 150000 Ukrainiens se trouvaient toujours en Pologne orientale lorsque fut décrétée la fin officielle des transferts de populations à l'été 1946. Dans un deuxième temps, les autorités polonaises, au prétexte d'une résistance nationaliste active au sein des foyers restants, organisèrent une nouvelle opération, dite « opération Wisła » qui consista, à partir du printemps 1947, à déporter tous les foyers ukrainiens des régions frontalières et à les disséminer dans les territoires récemment dé-germanisés de Poméranie et de Silésie. Ce dernier épisode tragique constitue le point de vue ultime à partir duquel est reconstituée l'histoire du transfert des Ukrainiens de Pologne et l'on ne s'étonnera pas, dès lors, de l'attention qui a été prioritairement portée sur le rôle des agents étatiques polonais dans cette histoire. Bien que la présence, en Pologne orientale, d'une administration soviétique ukrainienne chargée d'évacuer sa minorité soit un fait incontesté et bien que la complicité

1. Selon les estimations les plus communément admises, le bilan de ces différents affrontements serait de 80000 victimes polonaises (principalement en Volhynie) et de 20000 victimes ukrainiennes en Pologne.

2. L'organisation des nationalistes ukrainiens (OUN, Organizacija ukraïns 'kih nationalistiv) avait été fondée à Vienne en 1929 avec pour objectif de lutter contre l'oppression dont étaient victimes les Ukrainiens de Pologne en ayant recours à des pratiques terroristes. L'Armée insurrectionnelle ukrainienne (UPA, Ukraïns 'ka Povstans 'ka Armija), fut, elle, créée en Volhynie en 1943. Malgré leurs divergences au cours de la guerre, ces deux organisations se rapprochèrent à l'issue du conflit. L'Armée de l'intérieur (AK, Armja Krajowa) fut la plus grande organisation de résistance polonaise créée sous l'autorité du gouvernement polonais en exil. Ses dirigeants appelèrent à cesser son activité en janvier 1945, mais de nombreux groupes locaux poursuivirent leur action de résistance, notamment dans les régions orientales où divers accords furent passés avec les organisations ukrainiennes pour coordonner la lutte contre les tenants du régime communiste.

3. Pour une présentation critique de ces travaux et de la très riche bibliographie constituée sur le sujet, cf. Rafał Wnuk, «Recent Polish Historiography on Polish-Ukrainian Relations during World War II and its Aftermath », Inter Marium, Institute of East-Central Europe, 2004 (http:// tinyurl.com/czejjz). 
de cette administration dans la conduite des opérations de l'armée en 1945-1946 soit un fait largement reconnu, son rôle est néanmoins demeuré très partiellement exploré et évalué. Le constat peut en partie s'expliquer par le caractère parcellaire de l'ouverture des archives ukrainiennes concernant cette question et la mise à disposition récente du fonds de l'appareil ukrainien de Pologne dont les correspondances, les réunions internes permettent, aujourd'hui, de mieux comprendre quelles étaient les attentes, les enjeux formulés par les autorités de Kiev relativement au « retour » de la minorité ukrainienne dans la mère patrie. Longtemps considérés comme de simples exécutants des décisions prises au sommet de l'État soviétique, les dirigeants kiéviens ont, en réalité, conçu des objectifs spécifiques pour la république soviétique d'Ukraine dans la perspective de sa reconfiguration et de sa reconstruction aprèsguerre. Ceux-ci se sont donnés à voir précisément dans la période genèse des transferts de populations, soit à partir de l'été 1944 et jusqu'à la fin de la guerre. Cette première séquence, jugée peu problématique en raison des moindres affrontements n'a, pour cette raison notamment, pas beaucoup suscité l'attention. Or, elle éclaire de façon décisive les intentions et les agissements des agents ukrainiens à une époque où les régions frontalières échappent très largement au contrôle du nouveau pouvoir polonais. Les contradictions béantes entre la volonté d'initier un vaste mouvement de migrations spontanées vers l'Ukraine, les pratiques usitées pour ce faire et les moyens à disposition préfigurent, dès cette première année, la grande faillite du projet de transplantation pacifique de la minorité.

\section{La libération des territoires et le projet de Grande Ukraine}

Lors de la première annexion de l'Ukraine occidentale en 1939, l'argument selon lequel les Polonais, minoritaires dans cette région, n'avaient pas de légitimité à y exercer leur pouvoir comme État-nation tutélaire avait été présenté comme le fondement de l'entreprise de « libération » soviétique de la région. Il avait permis aux autorités de Kiev d'asseoir le processus d'annexion en jouant des vieux antagonismes nationaux. À travers la dénonciation de l'impérialisme polonais, la direction ukrainienne se posa en porte-parole des opprimés et donna libre cours au ressentiment multiséculaire des ruraux ukrainiens à l'encontre des Pan ou seigneurs polonais. En 1944, l'argumentaire anti-polonais fut repris avec force. Lors de l'offensive victorieuse de l'Armée rouge en Ukraine occidentale, la reprise en main des territoires fut d'abord placée sous le signe de la réunification du pays. Les actes symboliques de réappropriation nationale se multiplièrent à commencer par les changements de noms des principales villes occidentales, désormais « ukrainisées $»^{4}$. La volonté d'effacer

4. CDAGOU (Central'nij deržavnij arhiv gromads'kih ob’ednan' Ukraïny - Archives centrales d'État des organisations populaires d'Ukraine) f. 1, op. 23, d. 709, p. 4 sqq. : correspondance d'août 1944 entre Kalinin (Président du Soviet suprême de l'URSS) ou son adjoint Gorkin et Hruščev à propos d'un projet de décret soumis par ce dernier relatif au changement de nom d'un certain nombre de villes et de districts d'Ukraine occidentale telle que Tarnopol qui est alors rebaptisée Ternopol (et non Ternopil), Stanisławów qui est rebaptisée Stanislaviv. 
l'empreinte culturelle des anciens dominants allait de pair avec celle d'en chasser leurs représentants.

Au cours de l'été 1944, le gouvernement ukrainien préconisa avec force l'organisation de l'évacuation de la minorité polonaise vers la Pologne 5 et accueillit l'accord préparé à Moscou sur les transferts de populations comme le feu vert donné à l'entreprise d'épuration ethnique.

Différente fut en revanche la configuration qui présida à la question du devenir de la minorité ukrainienne de Pologne qui, au cours de cette même période préoccupa autant sinon davantage les dirigeants de Kiev. Cette préoccupation se manifesta dès l'été 1944 alors que 1'Armée rouge (1 ers fronts ukrainien et biélorusse), après avoir franchi le Bug, organisait son stationnement temporaire dans la région frontalière. Relayant les rapports des fronts sur la situation des Ukrainiens de Pologne, face à Stalin, Nikita Hruščev se fit l'ardent porte-parole de la minorité opprimée. Tandis qu'il n'avait pas fait grand cas des massacres dont les Polonais avaient été victimes en Ukraine, en particulier en Volhynie, mais avait insisté sur l'antisoviétisme viscéral de ces populations, le dirigeant ukrainien se faisait brusquement l'avocat des victimes ukrainiennes, effectivement sous le feu des représailles de leurs voisins polonais. Cette compassion pour le sort des « frères de sang » avait une visée stratégique, bientôt dévoilée, qui était d'étendre les frontières occidentales de l'Ukraine en y intégrant les régions de fortes concentrations ukrainiennes.

Tout au long de l'été 1944, les pétitions collectives des Ukrainiens de Pologne se multiplièrent, tantôt à l'adresse du maréchal Stalin, tantôt à celle du président Kalinin, tantôt encore à celle du dirigeant ukrainien, Hruščev. Rédigées sous la forme de véritables suppliques, ces pétitions faisaient part des violences exercées à l'encontre des Ukrainiens et qui, selon les auteurs, s'inscrivaient dans la continuité de la politique répressive conduite par le pouvoir polonais dans les années 1930. Rappelant que les signataires représentaient des communautés villageoises entières installées depuis de nombreuses générations dans leurs terres, les pétitions s'achevaient par la demande non pas de quitter ces territoires hostiles mais que ceux-ci soient rattachés à la patrie soviétique ukrainienne. Ainsi s'achevait de façon emblématique l'une d'entre elles : «humblement, intensément et fermement nous te demandons, Père, Maréchal, Camarade Stalin, de nous libérer de cette nouvelle servitude et de rattacher nos terres, depuis des siècles ukrainiennes, de la Holmščina ${ }^{6}$, à la mère patrie ukrainienne d'Union soviétique ${ }^{7}$. Alors que ces courriers collectifs s'amoncelaient dans les administrations soviétiques, Hruščev invoquait parallèlement la nécessité de

5. Cette position avait été clairement formulée à l'occasion de la « réévacuation » de Polonais d'Ukraine occidentale déportés dans les Komis en 1940 et qui avaient été autorisés par Stalin en juillet 1944 à s'installer dans les kolkhozes d'Ukraine centrale et orientale, sans que l'avis des autorités ukrainiennes n'ait été sollicité, ce qui entraîna leur réaction défavorable et leur proposition d'évacuer les Polonais considérés comme des «pilsudkistes » invétérés. Sur cette question, cf. GARF (Gosudarstvennyj arhiv Rossijskoj Federacii - Archives d'État de la Fédération de Russie), f. 5446, op. 46a , d. 280-261 et f. 5446, op. 47a, d. 254.

6. Terme désignant la région de Holm ou Chełm en polonais.

7. CDAGOU,f. 1 , op. 23, d. 790,1. 16. 
programmer la soviétisation de ces régions. Dans l'un de ses rapports à Stalin, il précisait ainsi qu'il convenait de « joindre à l'Ukraine soviétique les districts de Holm (Chełm), Grubešuv (Hrubieszów), Zamost' (Zamość), Tomašuv (Tomaszów), Jaroslav (Jarosław) et quelques autres points touchant aux régions indiquées. En réunissant l'ensemble de ces districts on pourra former la région de Holm avec pour centre administratif la ville de Holm $»^{8}$. Pour justifier ce projet, Hruščev s'appuyait non seulement sur les demandes adressées dans ce sens par les populations locales mais aussi sur le fait que cette région avait été autrefois rattachée à l'Empire russe. Une administration « spéciale » d'empire avait été en effet tardivement mise en place (1911) détachant la Holmščina de l'administration du royaume de Pologne et Hruščev légitimait désormais son plan sous la forme d'une réparation historique en faisant référence à une décision qui, à l'époque, avait été reçue en Pologne comme l'une des ultimes manifestations de l'impérialisme grand russe ${ }^{9}$.

La région de la Holmščina, située entre L'viv et Lublin en Pologne représentait l'une des zones stratégiques dans l'avancée des armées vers l'ouest et elle devint le centre de stationnement des fronts avant l'offensive décisive, entreprise fin janvier 1945, vers Berlin. La proposition de Hruščev fut amplement relayée par les commandants militaires de la région qui avaient organisé le « plébiscite spontané » des populations locales en faveur de leur rattachement à l'Ukraine soviétique et qui soutinrent fortement le projet de «Grande Ukraine ${ }^{10}$. Néanmoins le plan krouchtchevien ne fut pas retenu à Moscou. D'une certaine manière, l'accord du 9 septembre 1944 signé entre l'Ukraine et la Pologne pour procéder à l'évacuation réciproque de leurs minorités sanctionnait le désaveu de Stalin au projet de « Grande Ukraine ${ }^{11}$.

\section{L'organisation des transferts et le deuxième projet ukrainien}

Ce premier accord sur les transferts de populations ethniques de la période de sortie de guerre était, dans les textes, d'une transparence incontestable et d'un respect irréprochable des droits humains. Il stipulait en premier lieu le caractère volontaire

8. Rapport de Hruščev à Stalin du 20 juillet 1944, reproduit notamment par Volodimir Sergijčuk, Tragedija Ukraïnciv Pol'šči, Ternopil’ : Vidavnictvo Ternopil’, 1997, p. 65-70. La mention entre parenthèses des noms de villes en polonais (telles qu'elles sont restées) est de nous.

9. Theodore Weeks, Nation and State in Late Imperial Russia : Nationalism and Russification on the Western Frontier, 1863-1914, Dekalb : Northern Illinois University Press, 1996.

10. Les rapports réguliers des responsables de la direction politique du front ne manquèrent jamais de rappeler jusqu'au départ des armées de la région que les Ukrainiens de Holmščina et d'ailleurs espéraient toujours le rattachement de leurs terres à l'Ukraine. Cf. Jurij Slivka, Deportaciï zahidni zemli Ukraïny kincja 30-h počatku 50-h rr. Dokumenty, materialy, spogady u tr'oh tomah [Les déportations des terres occidentales de l'Ukraine, fin des années 30-début des années 50, Documents, matériaux, mémoires en trois tomes], t. I, 1939-1945, L' viv : Nacional’na Akademia nauk Ukraïny, 1996, p. 284-286, 309-311, 379-389, 399-406.

11. Nous n'avons trouvé aucune trace d'une quelconque réponse de Stalin à Hruščev, que ce soit dans les archives ou les travaux historiques de la période. L'accord polono-ukrainien est reproduit dans Slivka, Deportaciï..., p. 287 sqq. 
des départs. Les minorités, ukrainienne de Pologne et polonaise d'Ukraine étaient encouragées à regagner leur patrie respective mais ne devaient en aucun cas y être forcées. Les familles, candidates au départ, avaient le droit d'emporter leurs biens (dans la limite de deux tonnes par foyer) et étaient assurées d'obtenir dans leur future région d'installation un logement équivalent à celui laissé sur place. Elles étaient donc invitées à se faire enregistrer et à faire enregistrer leurs biens auprès d'une administration ad hoc. L'accord prévoyait en effet la mise en place de représentations nationales, polonaise en Ukraine et ukrainienne en Pologne, chargées, chacune, de superviser l'évacuation de ses nationaux. Un plénipotentiaire d'Ukraine fut nommé dès la mi-septembre 1944 pour créer une administration centrale ukrainienne à Lublin et organiser des agences locales dans tous les districts de forte concentration ukrainienne (il y en eut 15 durant la période concernée) ${ }^{12}$. En outre, pour garantir le caractère bilatéral de l'accord et prévenir tout contentieux, le texte prévoyait la nomination de représentants de l'autre partie qui seraient les interlocuteurs privilégiés de ces administrations et veilleraient à la bonne application de la procédure, notamment en confirmant par voie administrative tous les enregistrements effectués. Enfin, l'accord annonçait une prompte réalisation des transferts puisqu'il fixait la fin des opérations au 1er février 1945. Ce délai parfaitement irréaliste, qui allait être régulièrement amendé jusqu'en juin 1946, soulignait bien la volonté soviétique d'engager de vastes mouvements de populations dans le sillon tracé par les armées et d'établir de facto la frontière ethnique avant les grandes négociations internationales et bilatérales sur la nouvelle ligne de délimitation des États.

Comment ce cadre théorique de l'accord fut-il mis en application ? Les transferts polono-ukrainiens sont le plus souvent qualifiés par les historiens de «déportations », déportations d'un genre certes singulier puisqu'il s'agissait d'expatriations forcées et non, comme c'était jusqu'alors le cas en URSS, de déplacements massifs au sein du territoire soviétique ${ }^{13}$. Mais ce qualificatif se rapporte surtout à la période la plus étudiée et conflictuelle des transferts qui se déroula après la fin de la guerre. A-t-il été sous-entendu dans la conception même de ces déplacements de populations ? Peu après la signature de l'accord, le général Bulganin, dans

12. Au total, 375 personnes furent employées dans l'administration ukrainienne de Pologne au cours de ses deux années d'activité mais, compte tenu de la rotation du personnel, 250 en moyenne se trouvèrent sur le terrain. Plus de 200 avaient été nommées par le département des cadres du comité central du parti d'Ukraine. La très grande majorité des administrateurs étaient ukrainiens mais provenaient exclusivement d'Ukraine centrale et orientale (et principalement de Kiev), cf. CDAVOU (Central'nyj deržavnyj arhiv vyščyh orhaniv vlady ta upravlinnja Ukraïny - Archives centrales des organes suprêmes de l'État d'Ukraine), f. 4959, op. 1, d. 72.

13. Malgré l'interprétation somme toute assez nuancée de cette histoire que fit Eugeniusz Misiło dans son introduction à la publication de documents d'archives polonais sur les évacuations d'Ukrainiens (cf. Repatriacja czy deportacja. Przesiedlenie Ukraińców z Polski do USRR 1944-1946 [Rapatriement ou déportation. Le déplacement des Ukrainiens de Pologne vers l'Ukraine soviétique], 2 vol., Varsovie : Archiwum Ukrainskie, 1996-1999), les historiens se sont beaucoup référés à cet ouvrage pour considérer ces transferts comme des déportations (cf. notamment les contributions de G. Mazur et W. Serhiczuk pour ce qui est de l'historiographie polonaise et ukrainienne in Polska-Ukraina : trudne pytania, $\mathrm{n}^{\circ}$ 8, Varsovie : Karta, 2001, p. $15-57$ et $58-85$ ). 
ses instructions internes sur l'organisation concrète des transferts, rappelait fermement en préambule le caractère strictement volontaire des candidats au départ ${ }^{14}$. S'agissait-il donc là de pure démagogie ?

La position des autorités doit ici être envisagée par référence au deuxième objectif que la direction ukrainienne se fixa. En effet, dès le début de l'automne, un vaste plan de repeuplement de la minorité ukrainienne de Pologne fut conçu à Kiev . Prenant en considération le fait qu'il s'agissait d'une population à plus de $90 \%$ rurale, les autorités ukrainiennes envisagèrent d'envoyer massivement ces familles de paysans dans les kolkhozes d'Ukraine centrale et orientale qui avaient particulièrement souffert de la guerre et se trouvaient fortement démunis en forces laborieuses, en particulier masculines. Dans l'esprit des autorités, cependant, le transfert des hommes apparaissait aussi important que celui de leurs animaux, de leurs outils, voire d'une partie de leur grain. Le plan ne visait donc pas simplement à un repeuplement des fermes collectivisées mais tout autant à une redynamisation de l'agriculture ukrainienne par l'importation de l'ensemble des foyers de production. Lors de la première réunion de l'appareil ukrainien de Pologne, Koročenko, le secrétaire général du PC ukrainien qui s'était alors déplacé pour donner les instructions du gouvernement, ordonna fermement à ses agents d'inciter les candidats au départ à prendre le maximum de biens «qu'ils prennent donc la volaille, les animaux, le grain, mais nous ne pouvons soutenir ${ }^{15}$. Sans aucun doute, il faut prendre toute la volaille et les animaux [...]. En ce qui concerne l'inventaire, les charrues, les herses $[\ldots]$, prenez tout ${ }^{16}$.

Lors de la réunion qui se tint un mois plus tard, l'expert du gouvernement ukrainien présent réitéra en les précisant les besoins :

Il nous faut prendre le plus d'animaux possible. La majorité de la population évacuée vient chez nous dans le sud, c'est-à-dire là où il reste très peu d'animaux après l'occupation. Ici, il y a de très bonnes vaches hollandaises, très productives. Il y a des cas où les gens qui doivent être évacués tuent leurs animaux. Là, il faut conduire un travail de masse pour que les gens prennent avec eux leurs génisses et leurs chèvres. Et avec ce complément on va redresser les kolkhozes. C'est une tâche très importante et nous ne devons pas manquer ce moment. Beaucoup de paysans, sachant qu'ils devront donner leurs chevaux, préfèrent les vendre [...]. Chez nous, la situation est également difficile avec le charroi. Maintenant en ce qui concerne les cochons, les gens les tuent avant de partir, qu'ils les tuent, cela nous est égal. Pour les volailles je n'ai pas d'ordre précis. Vous savez que les Allemands ont apporté la maladie de la volaille. Cette maladie s'est répandue en Europe [...] et elle apparaît chez nous. C'est pourquoi

14. CDAGOU, f. 1 , op. 23 , d. 1240. N. Bulganin agissait alors en tant que représentant du sovnarkom de l'URSS auprès du PKWN.

15. Sous-entendant qu'en principe les paysans étant limités à deux tonnes par foyer, les administrateurs ne pouvaient officiellement apporter leur soutien au transfert de tous les animaux qui excédaient le poids autorisé.

16. CDAVOU, f. 4959, op. 1.1, d. 6, compte rendu de la réunion du plénipotentiaire d'Ukraine à Lublin, 23-24 octobre 1944,p. 18. 
il est recommandé que les paysans ne prennent pas trop de poules et qu'ils emportent plutôt les oies et les canards..$^{17}$

Quant à la question du grain, il était désormais ordonné que les foyers n'en prennent que la quantité nécessaire à leur temps de voyage et qu'ils laissent tout le reste de leur production agricole à l'Armée rouge ${ }^{18}$.

Autrement dit, plus qu'un simple déplacement de populations, les autorités ukrainiennes planifiaient un véritable transfert économique qui s'apparentait aux grandes opérations d'évacuations conduites dans l'urgence lors de l'invasion allemande au début de l'été 1941, à ceci près qu'il s'agissait désormais d'une évacuation conçue dans un contexte de sortie de guerre et de reconstruction économique du pays.

D'après la planification des transferts élaborée dans le cours de l'automne 1944, il était prévu que $70 \%$ des foyers ukrainiens de Pologne soient ainsi relocalisés dans les kolkhozes d'Ukraine centrale et orientale, les autres $30 \%$ devant être installés en Ukraine occidentale ${ }^{19}$. Autrement dit, les autorités prévoyaient bien, malgré le libre arbitre théoriquement laissé aux individus, de transférer l'ensemble de la minorité ukrainienne de Pologne. Néanmoins, compte tenu de l'objectif assigné, ces déplacements ne pouvaient se faire selon les pratiques bien expérimentées des agents soviétiques de déportations effectuées sous la contrainte directe et la violence. La réussite du plan de transfert dépendait au contraire de l'adhésion des individus au projet. Il supposait en effet que les foyers organisent consciencieusement leur départ, qu'ils prévoient le fourrage et le grain pour leurs animaux, qu'ils fassent un tri raisonné des instruments et outils les plus indispensables, qu'ils préparent au mieux leur chargement et leur voyage afin de pouvoir, à l'avenir, constituer pleinement ces nouvelles forces attendues pour relever l'économie agricole ukrainienne.

\section{Susciter l'adhésion, faire preuve de vigilance politique, trouver les moyens de persuasion}

L'impératif du départ « volontaire » des paysans ukrainiens plaçait les administrateurs devant l'objectif prioritaire du « travail de propagande », par l'organisation de meetings, l'envoi de « référents ${ }^{20}$ dans les villages, chargés de convaincre les

17. CDAVOU, f. 4959, op. 1.1, d. 6, compte rendu de la réunion du plénipotentiaire d'Ukraine à Lublin le 25 novembre 1944, p. 56.

18. Ibid., la question de la remise de la production à l'armée et l'organisation des réquisitions furent fixées dans cette période seulement.

19. CDAVOU, f. 4959, op. 1.1, d. 2, plan d'évacuation et de répartition de la population ukrainienne pour l'année 1944. Rappelons, cependant, qu'en dépit de la précision comptable, de la planification faite, le nombre d'Ukrainiens dénombré dans les régions orientales de la Pologne ne cessa d'augmenter durant toute la période considérée.

20. Comme l'expliquait l'adjoint au plénipotentiaire d'Ukraine à Lublin, le camarade Romaščenko, «le référent est le travailleur opérationnel fondamental de chaque appareil régional [...]. Il a à charge de conduire les explications concernant le travail dans les villages, il 
exploitants les plus influents de s'enregistrer afin de « faire bouger » l'ensemble de la communauté villageoise. Les résultats de la première campagne de propagande furent très inégaux et révélèrent surtout la très grande disparité des situations locales.

Les Ukrainiens de la région de Chełm furent les premiers à répondre positivement à l'appel ce qui, à vrai dire, n'avait rien d'étonnant. La concentration de l'Armée rouge dans cette région depuis l'été 1944 avait constitué un vecteur naturel de propagande. L'élan patriotique suscité par l'arrivée des libérateurs dans ce bastion de l'orthodoxie qu'était la Holmščina et le soutien, voire l'incitation témoignée par les autorités militaires au projet de Grande Ukraine défendu par Hruščev apparaissaient là comme les principaux fondements de l'incontestable popularité des Soviétiques ${ }^{21}$. D'autres facteurs, cependant, contribuaient à « l'enthousiasme » au départ vers la patrie, à commencer par la menace d'agression de la résistance polonaise $(\mathrm{AK})$, menace d'autant plus forte qu'une partie des ruraux ukrainiens avaient été placés par les Allemands dans des exploitations d'où avaient été chassés les paysans polonais ${ }^{22}$. Les rapports de l'administration ukrainienne au cours de l'automne et de l'hiver 1944 soulignaient immanquablement le « désir » des Ukrainiens de cette région d'être installés dans les kolkhozes d'Ukraine centrale parce que, soulignaient-ils, les gens souhaitaient se trouver loin des bandes nationalistes et avoir l'assurance du pain quotidien : « Là, soulignait l'un des administrateurs à propos de l'adhésion forte au projet de relocalisation, notre armée a bien travaillé, on leur a déjà tout dit $\gg^{23}$. Dans cette région également, la présence du clergé orthodoxe jouait, toujours selon les rapports, un grand rôle. Les prêtres venaient d'euxmêmes solliciter les représentants ukrainiens pour organiser le « retour » de leurs ouailles dans la mère patrie ${ }^{24}$. Début décembre, près de 30000 Ukrainiens sur les 34000 du district de Chełm s'étaient déjà fait enregistrer pour partir et le bilan comptable faisait également apparaître un fort taux d'enregistrement dans les districts adjacents 25 .

doit accueillir les requêtes, établir les registres des biens laissés, enregistrer la quantité de produits comestibles, aider les évacués à recevoir des conditions avantageuses de transports, organiser les transports et les conduire jusqu'au point [de frontière] prévu », CDAVOU, f. 4959, op. 9, d. 3, 1. 14, rapport à Hruščev du 6 novembre 1944.

21. Dans cette région également, l'implantation relativement forte du parti communiste dans l'entre-deux-guerres, toujours attestée durant la guerre par l'importance du mouvement partisan, doit également être prise en compte, cf. à ce propos Orest Subtelny, «Expulsion, Resettlement, Civil Strife : The Fate of Poland's Ukrainians, 1944-1947 », in Philipp Ther, Ana Siljak, eds., Redrawing Nations : Ethnic Cleansing in East-Central Europe, 1944-1948, Boston : Rowman et Littlefield, 2001,p. 157.

22. Le fait est régulièrement souligné par les responsables ukrainiens pour expliquer notamment les conflits rencontrés avec leur homologues polonais dans l'enregistrement des biens immobiliers des évacués, mais les rapports ne contiennent aucune évaluation, même approximative du phénomène.

23. CDAVOU, f. 4959, op. 1.1, d. 6, doc. cit, p. 12.

24. Cf. notamment le rapport du général Šatilov à ce propos du 9 novembre 1944, CDAGOU, f. 1 , op. 23 , d. $790,1.81-86$

25. CDAVOU, f. 4959, op. 1.1, d. 35, p. 79-85, rapport de Kal’nenko du 31 décembre 1944. 
Cette première grande vague de candidats volontaires au départ avait de quoi conforter le projet de transfert économique car les districts concernés étaient loin d'être si pauvres qu'on a pu souvent l'affirmer et les premiers convois qui partirent furent parmi les plus « rentables » de la période, eu égard, notamment, à la proportion d'animaux par foyer ${ }^{26}$. D'autre part, l'administration ukrainienne de Pologne, placée sous l'injonction de réaliser le plus rapidement possible les transferts, pouvait, par ces premiers résultats, attester son efficacité. Cependant, la perspective de déplacer ces dizaines de milliers de paysans vers l'Ukraine soviétique posait la question de leur loyauté politique, malgré les démonstrations d'enthousiasme qui s'étaient manifestées au cours de la campagne d'enregistrement. Lors d'une réunion de la commission ukraino-polonaise à Lublin fin novembre, les représentants polonais avaient abordé cette question, soulignant que nombre de candidats cherchaient par leur départ à fuir le risque de représailles liées à leur collaboration passée avec les Allemands. La migration vers l'Ukraine était ainsi suspectée d'être, pour certains, une stratégie visant à éviter la dénonciation des voisins et à effacer, dans l'anonymat des groupes de paysans migrants, un passé condamnable. Ce problème mettait l'administration ukrainienne devant un certain dilemme car, si la nécessité d'effectuer une épuration politique apparaissait comme une évidence, elle constituait tout autant une nuisance à la popularité de l'opération de transferts. Alors que les nationalistes ukrainiens commençaient à développer une forte contre-propagande, insinuant, notamment, que les populations déplacées seraient envoyées en Sibérie, la conduite d'une « vérification » politique parmi les candidats au départ ne pourrait que conforter l'argumentaire des «bandes nationalistes ». Finalement la tactique choisie par l'administration ukrainienne fut définie lors d'une concertation avec tous les agents régionaux de l'appareil par l'adjoint du plénipotentiaire (et vicecommissaire du NKVD d'Ukraine), Kal'nenko. Celui-ci, en effet, aborda bientôt le problème de la «vigilance politique ». Il était bien évident, selon ce responsable, que les candidats au départ n'étaient pas forcément convaincus de la bonne vie dans les kolkhozes et qu'ils cherchaient plutôt :

à éviter [par leur départ] d'être punis pour leur collaboration avec les Allemands. Mais ces gens-là doivent répondre de leurs crimes et nous devons les identifier. Donc, apprenez, Camarades, qu'ils nous faut rapidement vérifier les évacués [...]. S'ils veulent partir, on les laisse partir mais on les connaît. À Holm (Chełm), il y a une coopérative qui est tout entière aux mains d'une organisation de nationalistes

26. L'explication donnée des premiers départs volontaires d'Ukrainiens font en effet valoir que la plupart n'avait rien à perdre, que la région de Chełm avait été particulièrement dévastée par la guerre (cf. notamment Roman Drozd, Droga na Zachód. Osadnictwo ludności ukraińskiej na ziemach zachodnich i pótnocnych Polski w ramach akcji «Wisła » [La route vers l'ouest. L'installation de la population ukrainienne dans les territoires de l'ouest et du nord de la Pologne dans le cadre de l'opération Wisła [Vistule]], Varsovie : Tyrsa, 1997, p. 21 sqq. ; Subtelny, «Expulsion, Resettlement, Civil Strife...»). Or, la relative abondance de la Holmščina fut bien mise en évidence dans l'état des lieux dressé par les responsables locaux (cf. CDAVOU, f. 4959, op. 1.1, d. 6, p. 35 sqq.). Comme le montra cette réunion de l'appareil, était considéré comme paysan riche, tout foyer possédant au moins une tête de bétail et très riche celui qui possédait à la fois une vache et un cheval, ce qui était le cas de plus de $50 \%$ des foyers de la Holmščina. 
ukrainiens, mais cette coopérative fait tout pour partir [...]. Nous avons décidé de la laisser partir, qu'ils partent car sinon cela aura une mauvaise influence sur la masse des gens qui nous intéressent, qu'ils partent donc, mais nous devons les connaître. Comme vous le savez notre personnel opérationnel n'est pas nombreux mais le communiste c'est un tchékiste [...]. Ce n'est pas simple d'envoyer les gens dans les kolkhozes, beaucoup fuient [la Pologne]. Vous savez quelle est la situation dans nos kolkhozes, il n'y a pratiquement pas d'hommes là-bas et nous y envoyons $40 \%$ d'hommes dont des contre-révolutionnaires et des éléments nationalistes. C'est que nous en envoyons 300000 dans cinq districts, ce qui fait un grand pourcentage par district alors que dans la plupart des kolkhozes il n'y a que des femmes [...] et sur 100 foyers [à déplacer] il y en a au moins 15-20 qui sont défectueux..$^{27}$

Ainsi la tactique consistait à repérer les « éléments défectueux » de façon à pouvoir les arrêter une fois passée la frontière. Bientôt, cependant, l'existence des « points de filtration » prévus pour la vérification politique des évacués fut connue des candidats au départ. Comme le souligna le responsable du district de Lubaczów, certains foyers évacués avaient été témoins de l'existence d'un camp au point de frontière et peu après, plus personne ne voulait se faire enregistrer pour partir ${ }^{28}$.

À la veille des premiers grands départs d'Ukrainiens, en cette fin 1944, la vérification politique des évacués se révéla, cependant, un problème mineur pour les autorités qui jugeaient bien plus préoccupante la situation des districts se trouvant au sud et sud-ouest au long de la nouvelle ligne frontalière, hors du rayon de stationnement des armées. Dès l'automne, les rapports militaires avaient souligné la forte résistance manifestée par la population de cette zone à la perspective d'un transfert vers l'Ukraine et cette résistance s'expliquait, selon eux, par l'importante agitation des bandes nationalistes ukrainiennes. De fait, ces régions situées à l'écart des grandes zones de front étaient en partie des régions montagneuses bordant la frontière slovaque. Elles se présentaient stratégiquement comme des zones de refuge (voire de repli en Slovaquie) pour la préparation d'actions d'envergure contre le pouvoir soviétique. Ce facteur était cependant peu relevé. En revanche, les commandants militaires mettaient l'accent sur la spécificité culturelle et religieuse des Ukrainiens du sud-est de la Pologne. Plus les rapports s'amoncelaient et plus cette différence soulignée était présentée comme la source du clivage entre «bons » et «mauvais »Ukrainiens : «Dans sa majorité, le clergé orthodoxe soutient l'évacuation des Ukrainiens [...] alors que les gréco-catholiques n'y sont pas favorables ${ }^{29}$, affirmait un responsable en fournissant à l'appui de cette déclaration nombre d'arguments sur le comportement des prêtres des deux confessions. Outre les distinctions religieuses, le rôle historique des anciens partages du territoire

27. CDAVOU, f. 4959 , op. 1.1, d. 6,1. 57-58.

28. CDAVOU, f. 4959, op. 1.1, d. 7, compte rendu de la réunion du plénipotentiaire d'Ukraine à Lublin du 20 janvier 1945, p. 14. Sur la mise en place des points de filtration, cf. Ivan Bilas, Represivno-karal'na sistema v Ukraini 1917-1953 [Le système répressif et punitif en Ukraine], Kiev-L'viv : Vijs'ko Ukraini, 1994, p. 208 sqq.

29. Slivka, Deportaciï..., p. 386 (rapport du département politique du 1er front d'Ukraine du 28 décembre 1944). 
polonais entre les Empires russe et austro-hongrois était également interprété comme un facteur décisif dans le sentiment pro-soviétique des uns et la résistance des autres :

la différence d'attitude s'explique par le fait que les territoires du nord étaient russes jusqu'en 1918 et que la majorité de la population considère la Russie et l'URSS comme sa patrie tandis que les territoires du sud, pour avoir appartenu à l'Empire austro-hongrois et à la Pologne, ne se sentent pas concernés. ${ }^{30}$

Cette « explication» des disparités politiques par le recours à l'histoire longue et partagée de la Pologne fut essentiellement formulée par les autorités militaires et allait devenir, par la suite, le leitmotiv invoqué par l'administration ukrainienne pour justifier la résistance rencontrée au sud. Les appareils régionaux ne furent constitués que tardivement dans la région, soit fin 1944, et ils y arrivèrent animés de forts préjugés contre les « Galiciens gréco-catholiques » ${ }^{31}$. Pourtant des motifs infiniment plus pragmatiques et récents expliquaient, dans certains cas, la force du sentiment anti-soviétique des locaux. Ainsi, dans le premier exposé de la situation qu'il fit devant ses supérieurs, le responsable du district de Przemysł (réputé pour être l'un des bastions de la résistance ukrainienne) distinguait la situation relativement calme des terres de la rive gauche de la rivière San de celle des terres de la rive droite, « qui étaient ravagées par le banditisme ». Pour ajouter à la suite que cette rive droite avait été rattachée à l'URSS en 193932. En définitive, le responsable régional déplorait l'absence de toute autorité polonaise et le fait que les Ukrainiens, objectivement majoritaires dans le territoire en question, étaient totalement maîtres des lieux. De la même manière, le responsable du district de Lesko soulignait que sa «principale difficulté était qu'il n'y avait pas de Polonais dans la région. Il serait nécessaire, poursuivait-il, que les Ukrainiens voient que leur terre est en fait aux mains des Polonais ${ }^{33}$. Face aux résistances des districts du sud, Podgornyj, le plénipotentiaire d'Ukraine préconisa différentes tactiques pour entraîner le départ de la population. La première consistait à engager les autorités polonaises à exercer des «pressions économiques par les taxes et les impôts ${ }^{34}$. En second lieu Podgornyj comptait solliciter les autorités polonaises pour qu'elles organisent le travail forcé des Ukrainiens : «pour la construction des routes et des ponts il faut

30. Ibid., p. 403, rapport du département politique du 1er front d'Ukraine du 9 janvier 1945.

31. Notons cependant qu'en reprenant le discours des militaires, les administrateurs ukrainiens n'avaient que de très faibles connaissances de ces spécificités historiques. Ainsi l'adjoint au plénipotentiaire d'Ukraine expliquait que l'uniatisme «c'est quelque chose (eto čto-to) entre l'orthodoxie et le catholicisme avec une grande supériorité de l'influence catholique » (CDAGOU, f.1, op. 23/, d. 1465, 1. 5, rapport de Romaščenko du 19 janvier 1945).

32. En effet lors du partage de la région effectué dans le cadre du pacte germano-soviétique de 1939, la frontière germano-soviétique passait dans la ville même de Przemysł.

33. CDAVOU, f. 4959, op. 1.1, d. 7, compte rendu de la réunion du plénipotentiaire d'Ukraine à Lublin du 14 avril 1945, p. 27.

34. CDAVOU, f. 4959, op. 1.1, d. 7, réunion de l'appareil central à Lublin du 20 janvier 1945, p. 13. 
évidemment qu'ils mobilisent la population ukrainienne et attendent le moment le plus favorable pour ce faire ${ }^{35}$. Enfin, s'agissant de la «liquidation des bandes », le plénipotentiaire rappelait que cette tâche devait être effectuée par l'armée polonaise. Une déclaration qui allait devenir le leitmotiv de l'administration ukrainienne de Lublin face à ses interlocuteurs polonais et qui allait effectivement conduire à l'intervention de l'armée, mais à partir de septembre 1945, une fois la guerre achevée. En d'autres termes, les solutions préconisées par le représentant ukrainien visaient à déléguer toutes les mesures coercitives au pouvoir polonais de façon, notamment, à ne pas ternir l'image des Soviétiques présents en Pologne. D'autre part, en ce début 1945, l'administration ukrainienne réactiva une stratégie de « lutte de classes » qui faisait directement écho aux résolutions prises par le CC du parti ukrainien relativement aux koulaks considérés comme les alliés objectifs « des bandes de nationalistes ukraino-allemands » sévissant en Ukraine occidentale $^{36}$. S' adressant aux responsables locaux, Podgornyj martelait ainsi :

Vous vous trompez en vous orientant vers les instituteurs, les prêtres et les koulaks. Ne cherchez pas d'appui de ce côté-là. Il faut vous appuyer sur les pauvres et la couche moyenne de la population. ${ }^{37}$

À l'exposé du responsable du district de Jarosław qui rencontrait un échec patent à populariser l'idée du retour chez les Ukrainiens, il fut répondu :

Vous avez basé votre analyse d'après l'opinion de la moyenne intelligentsia [en allant] directement chez l'instituteur ou le prêtre [...] mais vous ne vous êtes pas approché du paysan pauvre, le vrai représentant du peuple et, pour nous, c'est très important [...]. Il faut prendre 10-15 foyers, qu'ils s'enregistrent et influencent alors les autres. ${ }^{38}$

Si les administrateurs ukrainiens s'intéressaient aux riches exploitants qui allaient avec leur bétail redresser les kolkhozes, ils comptaient avec une certaine candeur sur les pauvres pour, telle une courroie mécanique, entraîner le mouvement. Cette tactique se révéla d'autant plus vaine que les mouvements de départ furent bientôt contrariés par l'absence de trains.

35. Ibid. Rappelons que la «mobilisation obligatoire» pour la reconstruction des infrastructures fut aussi une pratique largement utilisée en Ukraine occidentale dès la fin de l'hiver 1944 pour lutter contre la résistance de l'OUN et de l'UPA. Cf. par exemple, le décret de mars 44 du Comité d'état à la défense (GKO) sur les mesures spéciales à prendre en Ukraine occidentale, in Volodimir Sergijčuk, Desjat' buremnih lit. Zahidnoukrains'ki zemli u 1944-1953 rr. Novi dokumenti i materiali [Une décennie tempétueuse. Les terres d'Ukraine occidentales dans les années 1944-1953], Kiev : vid. Dniepro, 1998, p. 53-54.

36. Ibid., p. 195-201, décret du 10 janvier 1945.

37. CDAVOU, f. 4959, op. 1.1, d. 7, réunion de l'appareil central à Lublin du 20 janvier 1945, p. 13.

38. Ibid., p. 7. 


\section{Quand Kafka s'en mêle}

La précocité de la planification des opérations de transferts relativement à la situation du front de l'Est s'exprima dès l'automne 1944 par d'importantes difficultés logistiques qui, au rythme de l'avancée de l'Armée rouge vers Berlin, allaient se transformer en véritables obstacles à la réalisation du plan. La première difficulté, ou la plus manifeste, fut le problème du transport. Au début, cela concernait moins la mise à disponibilité des trains (bien que les wagons demandés ne furent jamais obtenus en nombre suffisant ${ }^{39}$ ) pour transporter les foyers vers l'Ukraine, que l'acheminement des gens vers les gares. L'administration ukrainienne avait en effet compté sur la proximité de l'Armée rouge pour pouvoir bénéficier de ses engins motorisés, ce qu'elle n'obtint qu'au compte-gouttes. Or l'acheminement des foyers vers les gares (ou ce qui en tenait lieu car, dans ces régions rurales, il ne s'agissait bien souvent que d'un point d'arrêt, tout juste signalé, le long de la voie ferrée) représentait une tâche d'envergure. L'incitation faite par les agents ukrainiens aux exploitants à prendre le maximum de biens et à emporter tous leurs animaux transformait le déplacement d'un village en un vaste convoi d'hommes, de femmes et d'enfants mais aussi de vaches, d'oies, d'outils, de fourrage et de matelas... Faute de camions, les transports furent généralement organisés en charrettes (ou télègues) et, compte tenu des masses à transporter, celles des exploitants ne suffisaient pas. La nécessité de procéder à la réquisition des charrettes et des chevaux des voisins polonais devait incomber aux pouvoirs locaux polonais qui, dans bien des cas, n'avaient pas encore été constitués ou n'étaient présents qu'à titre symbolique. Il n'existait en guise de pouvoirs que des milices improvisées à l'échelle locale ou rapidement mises en place par les groupes d'anciens partisans. Autant dire que la plupart des villages étaient livrés à eux-mêmes. L'absence d'autorités municipales allait grandement se faire sentir dans le climat de sauvagerie croissante qui se manifesta dès le départ de l'armée.

Bien que la question générale des transports releva, dès le début, du registre de la pénurie, elle devint le problème principal à partir de février 1945. Les premiers convois vers l'Ukraine avaient pu être jusque-là, tant bien que mal acheminés. Près de 80000 Ukrainiens avaient été évacués entre le 15 novembre 1944 et le 20 février 1945 ce qui attestait du caractère effectif de l'évacuation ${ }^{40}$. Mais la carence de transports empira brusquement lors du départ de l'armée vers l'ouest. La priorité donnée aux besoins du front priva les régions frontalières de trains. Cette pénurie fut d'autant plus aiguë que s'amorçaient, en URSS même, les grandes réévacuations des populations et des industries qui avaient été déplacées dans l'urgence au moment de l'invasion allemande. Les confins polono-ukrainiens se trouvèrent ainsi dans le creux des deux très vastes mouvements d'hommes et de matériel qui, d'est en ouest de l'URSS et de l'Europe, requéraient l'essentiel du

39. CDAVOU, f. 4959, op. 9, d. 3, Bilan sur les évacuations des Ukrainiens de Pologne, déc. 1946, p. 256 sqq.

40. Rapport de Podgornyj du 2 mars 1945, Slivka, Deportaciï...,p. 435-447. 
parc ferroviaire disponible. L'injonction à poursuivre l'évacuation n'avait pas été pour autant mise en sourdine. Au contraire. L'amendement accordé à la date butoir de la fin des opérations (initialement fixée au $1^{\text {er }}$ février et repoussée au $1^{\mathrm{er}}$ mai) fut assorti d'un appel renouvelé à la vigilance politique des cadres pour achever les transferts dans les nouveaux délais impartis. La situation devint alors véritablement kafkaïenne : comment faire bouger (dvinut) les hommes et les animaux sans transports ? Le mode de gouvernance soviétique qui consistait à fixer les objectifs dans l'ignorance quasi délibérée des moyens nécessaires pour les atteindre avait, à vrai dire, déjà une longue histoire, en particulier dans les pratiques de déportation, avec la cohorte de désastres humains qu'une telle anticipation pouvait entraîner ${ }^{41}$. En l'occurrence, le désastre ne fut pas évité.

À défaut de trains, les agents de l'administration continuèrent à acheminer les familles et les biens vers les gares dans l'attente hypothétique de l'arrivée des convois. De très vastes campements s'improvisèrent le long des voies ferrées au cours de cet hiver particulièrement rude. Des milliers de familles se retrouvèrent, de fait, quasiment abandonnées dans la neige, sans toit et, le temps passant, sans vivres, sans fourrage ni grain pour leurs animaux. Les responsables ukrainiens égrenèrent immanquablement leurs rapports de nombreux cas critiques liés au stationnement interminable des foyers en attente de trains, soulignant surtout l'impopularité croissante des évacuations qui en résultait parmi la population non encore enregistrée au départ. De nombreuses familles inscrites commençaient à se désister. Ainsi, début mars près de 500 foyers renoncèrent à partir ${ }^{42}$. Dans le style comptable propre aux administrateurs soviétiques, Podgornyj signalait aussi 367 cas d'agressions répertoriés à l'encontre des «partants » qui s'était traduits par 52 meurtres, 350 chevaux volés, etc. ${ }^{43}$

En effet, la vulnérabilité des foyers stationnés le long des voies ne tenait pas seulement aux conditions critiques dans lesquelles ils survivaient mais aussi au fait qu'ils étaient devenus, dans biens des cas, la proie des bandes de pillards et de « nationalistes » qui sévissaient dans toute la zone frontalière.

\section{Contrées sauvages}

À partir de l'été 1944, lors de la libération des territoires de l'Ukraine occidentale puis de la Pologne orientale, les autorités soviétiques commencèrent à se concentrer prioritairement sur la liquidation des «bandes ». Dans cette période, l'ennemi, tel qu'il ressort des rapports, est clairement nommé. De ses tournées régulières dans les régions d'Ukraine occidentale (oblasts de Volhynie, Rovno, L'viv, Tarnopol),

41. Cf. l'excellente démonstration de ce phénomène par Nicolas Werth, L'île aux cannibales. 1933, une déportation-abandon en Sibérie, P. : Perrin, 2006.

42. Slivka, Deportaciï..., p. 342. et cf. sur toutes ces situations critiques d'attente les descriptions faites par les responsables régionaux, CDAVOU, f. 4959, op. 9, d. 3.

43. Ibid., p. 345. 
Hruščev rapporte à Stalin les évaluations faites de la présence des «nationalistes ukraino-allemands $»^{44}$. En Pologne orientale, les commissaires politiques des fronts consignent principalement les attaques des foyers ukrainiens par les Akovcy (membres de l'AK) et les «réactionnaires » polonais dans un contexte, il est vrai, alors spécifique, puisque ces rapports concernent essentiellement la Holmščina et accompagnent le «plébiscite spontané » des minorités pour le rattachement de cette région à l'Ukraine ${ }^{45}$. Peu après, au début de l'automne, la géographie des résistances armées se précise avec la mention, de plus en plus fréquente, de l'agissement des Ouncy, des banderovcy ou des bandes de l'UPA, surtout dans les districts situés au sud de la région de $\mathrm{Chełm}^{46}$. La consignation des attaques et agressions de ces bandes commence dès lors à constituer la chronique des événements ordinaires des régions frontalières sans que soient pour autant effectuées de quelconques évaluations des forces adverses ni qu'elles soient clairement identifiées. Sous la plume des responsables régionaux de l'administration ukrainienne de Pologne, banderovcy, bandes de l'UPA, bandes nationalistes sont le plus souvent synonymes (ce dernier terme pouvant s'appliquer aussi bien aux Ukrainiens qu'aux Polonais). À mesure que s'organise l'enregistrement des candidats à l'évacuation vers l'Ukraine, les actions dites de sabotage des opérations de transferts se multiplient. Les principaux visés sont les foyers de volontaires au départ. «Il ne se passe pas une nuit où nos paysans ne soient pas volés quand il n'y a pas des cas de meurtres », signale le responsable de Chełm à propos des agressions commises à leur égard ${ }^{47}$. Plus que les assassinats cependant, les raids organisés contre les « traitres » se caractérisent par le pillage des maisons, la prise du bétail et des chevaux, voire, pour finir, l'incendie des fermes. Comme le signalaient les victimes, leurs agresseurs s'emparaient des biens en proclamant que les évacués en seraient de toutes façons dépouillés lors du passage des frontières. « Ils nous ont dit que les soviets n'allaient pas nous conduire dans les kolkhozes mais nous envoyer en Sibérie », étaient des propos très communément rapportés dans la narration des agressions ${ }^{48}$. Dans le cas où les foyers étaient dépouillés par les bandes polonaises, il était fréquent que celles-ci annoncent : « vous partirez en Ukraine, mais vous y partirez nus ». Certains agresseurs commentaient : «les nôtres arrivent d'Ukraine seulement avec ce qu'ils ont sur le dos et vous partirez de la même manière ${ }^{49}$. Il est vrai qu'à partir de la fin

44. Sergijčuk, Desjat' buremnih lit..., p. 18-30, 33-35, 42-52, 63-80, 91-94, les rapports de Hruščev et des différents responsables régionaux du parti (obkomy) sur la situation politique des régions d'Ukraine occidentale.

45. Cf. les nombreux rapports de cette période in CDAGOU, f. 1, op. 23, d. 790.

46. Cf. les rapports militaires cités supra, et les rapports du plénipotentiaire, en particulier CDAVOU, f. 4959, op. 9, d. 3.

47. CDAVOU, f. 4959 , op. 1.1, d. 6 , compte rendu de la réunion de la commission mixte du 26 novembre 1944,p. 76.

48. Rapport de Podgornyj à Hruščev et Koročenko du 24 novembre 1944, CDAGOU, f.1, op. 23 , d. 790

49. Rapport du département politique du front, 28 décembre 1944, Slivka, Deportaciï..., p. 383. 
1944, les premiers convois de Polonais d'Ukraine arrivaient, n'ayant pu emporter avec eux que le strict minimum. À l'encontre du principe de réciprocité des accords passés, des obstacles administratifs au transport de leurs biens et leurs animaux avaient été posés, forçant les évacués à les abandonner sur place ${ }^{50}$. Cette situation ne faisait qu'accroître le ressentiment anti-ukrainien et les rapports de l'administration ukrainienne de Pologne insistaient toujours davantage sur la complicité, dans les opérations de pillage, des milices locales polonaises « infestées » par l'AK. Cependant, aux côtés des acronymes, AK, OUN, UPA, les agents évoquaient partout le banditisme, terme générique dominant de cette période. Lors de la réunion de la commission polono-ukrainienne de novembre 1944, il n'était plus question que de la lutte contre le fléau du banditisme, reconnu comme un phénomène majeur dans les régions frontalières de la Pologne. Toutes les agressions mentionnées dans cette concertation furent alors principalement assimilées à de vastes entreprises de pillages ${ }^{51}$. S'agissait-il, dans ce contexte bilatéral, d'éviter d'avoir à distinguer qui des «nationalistes » ukrainiens ou des « réactionnaires » polonais étaient les plus impliqués dans ces exactions ou plus simplement de rendre compte de la réalité du climat de prédation ambiante ? Toujours est-il que les représentants des deux parties n'abordèrent eux-mêmes que les différends d'ordre matériel liés notamment à l'évaluation et à l'enregistrement des biens des évacués, comme si l'essentiel des enjeux portaient non pas sur les milliers de foyers à déplacer mais sur leurs possessions. Cette remarque caractérise aussi la correspondance échangée entre les deux administrations où dominent les questions de spoliations, de rétentions, de détournement des biens des évacués ${ }^{52}$. Compte tenu de l'objectif fixé par le gouvernement ukrainien d'un véritable transfert économique des forces productives hommes, animaux et, partiellement, grain - cette préoccupation n'a pas lieu d'étonner. Mais n'a-t-elle pas contribué à alimenter les pratiques de prédation en présentant les agents de l'État eux-mêmes comme des prédateurs, engageant les évacués « à prendre tout ce qu'ils pouvaient »?

50. L'accord polono-ukrainien stipulait (art. 5) que, vu l'indigence de la situation en Pologne, la partie ukrainienne fournirait l'ensemble des transports nécessaires pour les évacuations. Lors de la réunion mixte des administrateurs polonais d'Ukraine avec leurs homologues (16 novembre 1944 à L'vov), ceux-ci imposèrent la signature d'une nouvelle instruction conditionnant le transport des biens des évacués à la disponibilité suffisante de wagons (CDAGOU, f. 1, op. 23, d. 790,1. 104 sqq.). Cette instruction fut immédiatement et systématiquement mise en application créant confusion et conflits multiples, cf. notamment, AAN (Archiwum Akt Nowych - Archives des documents contemporains), f. 524, syg. 28, p. 76-78, 100-103, 15658, 198 sqq., rapports de décembre 1944 des responsables polonais en Ukraine concernant la rétention des biens des Polonais et en particulier des animaux). Comme le signalait l'administrateur en chef de la planification des « rapatriements » des Polonais d'Ukraine, « il est fondamental que les gens prennent avec eux leurs animaux. Il faut se battre pour chaque tête de bétail [...] nous envoyons les gens dans des territoires vides », AAN, f. 524, sygn. 9, courrier de W. Wolski du 3 mars 1945.

51. f. 4959 , op. 1.1, d. 6 , compte rendu de la réunion de la commission mixte du 26 novembre 1944, doc. cit.

52. Cf. notamment, CDAVOU, f. 4959, op. 2, d. 1 ; f. 4959, op. 1.1, d. 1719-20, AAN, f. 524, sygn. 9-21-22, f. 526 sygn. 160 (A), 162 (A). 
La minorité ukrainienne de Pologne se trouvait prise dans l'étau de multiples pressions adverses. Les candidats à l'évacuation devaient remettre l'essentiel de leur récolte à l'Armée rouge. L'administration ukrainienne attendait de ses homologues polonais l'accroissement des « pressions économiques » pour favoriser le départ des récalcitrants, ce que les pouvoirs locaux polonais s'empressèrent de mettre en œuvre de manière d'autant plus décomplexée qu'ils étaient témoins du dépouillement dans lequel se trouvaient les Polonais tout juste évacués d'Ukraine. Les bandes, de leur côté, sévissaient soit pour punir les traîtres soit pour exiger le dû de leur lutte contre l'installation des «bolcheviks ${ }^{53}$. Au cœur de ce cercle infernal, les biens étaient devenus l'enjeu de toutes les rivalités. Tout était bon à prendre parce que tout manquait. Les autorités polonaises dénonçaient le fait que les foyers partants emportent avec eux jusqu'aux châssis des fenêtres de leurs maisons ${ }^{54}$, les responsables ukrainiens tentaient, eux, de les empêcher de vendre leur grain au marché noir pour acheter des chaussures ${ }^{55} \ldots$ L'état extrême de la pénurie peut être encore suggéré dans les requêtes répétées des plus privilégiés qu'étaient les agents de l'administration ukrainienne de Pologne, lesquels exigèrent à cor et à cri, mais en vain, que leur soient fournies des bottes et des manteaux chauds pour parcourir à pied dans la neige de longues distances entre les villages, faute de tout moyen de transport ${ }^{56}$. Hruščev lui-même, engagea une correspondance de plusieurs mois avec Berija afin d'obtenir une ligne téléphonique entre Kiev et Lublin de façon à pouvoir suivre la marche des évacuations des Ukrainiens de Pologne...

\section{Aveu de faillite}

Malgré le chaos et l'anarchie ambiante, renforcée à partir de février 1945 par le départ des troupes vers l'ouest, les convois d'Ukrainiens étaient peu à peu acheminés vers les kolkhozes d'Ukraine centrale, dans les régions de Dniepropetrovsk, Zaporože, Nikolaev, etc., dans des conditions très éprouvantes, liées non seulement

53. Comme dans toute l'histoire des résistances durant la guerre, qu'il s'agisse des mouvements de partisans ou des résistances nationales, la question se pose de savoir dans quelle mesure les populations civiles étaient complices ou otages des groupes armés. Dans les rapports des agents ukrainiens, elles apparaissent tantôt comme alliées de la guerilla, tantôt comme terrorisées par les bandes, sans que soient généralement fournis d'indices concrets sur lesquels fonder ces jugements. À ce sujet, cf. également l'article de Nathalie Moine dans ce numéro.

54. Fait rapporté avec indignation par Wolski lors de la réunion de la commission mixte de novembre 1944 (doc. cit.).

55. Fait rapporté par le responsable de Zamość lors de la réunion du plénipotentiaire du 2526 novembre 1944 (doc. cit.), et très souvent réitéré dans les rapports de l'ensemble de la période d'évacuation.

56. Dès cette même réunion de novembre, l'un des responsables régionaux haussa le ton en précisant que les référents n'avaient « pas besoin d'uniformes diplomatiques mais plutôt de vêtements chauds et de bottes ». Il s'avéra qu'à la énième répétition de cette requête, les commandes ne pouvaient être effectuées que via un département ad hoc du comité d'État à la Défense de Moscou... 
à la durée du voyage mais aussi à l'état des wagons, souvent à ciel ouvert, sans chauffage ni aucune autre commodité57. L'épreuve la plus redoutable était cependant à venir. En effet, les évacués arrivèrent dans des exploitations collectives totalement délabrées et se trouvèrent confrontés à des kolkhoziens qui, la plupart du temps, n'avaient même pas été prévenus de leur arrivée. L'absence de tout « travail de masse » mené à l'égard des kolkhoziens censés accueillir les évacués, de toute information fournie aux évacués eux-mêmes sur leur contribution attendue au relèvement des exploitations et de toute préparation matérielle d'accueil, eurent des conséquences désastreuses dans les face-à-face impromptus entre les locaux et les déplacés. Ceux-ci furent appréhendés comme des étrangers, souvent comme des «Polaks » et traités de «koulaks » par référence aux formidables richesses que constituaient alors la possession d'une vache et de quelques volailles au sein d'un foyer ${ }^{58}$. L'animosité s'avéra réciproque. Les soviets de village ne firent aucun effort ne serait-ce que pour loger de nouveaux arrivants et ceux-ci manifestèrent presque instantanément leur hostilité à la vie collective des kolkhozes ${ }^{59}$. L'état de délabrement des exploitations ajouté à l'évidence d'être mal venu, suscita une nouvelle idée fixe : revenir chez soi, dans les territoires orientaux de la Pologne d'où il aurait fallu ne jamais partir en dépit de l'insécurité ambiante. Les mouvements de retour clandestins ne nous sont connus qu'à travers leurs échecs ${ }^{60}$. À partir du printemps 1945 se multiplient les dénonciations de la corruption des agents polonais d'Ukraine, chargés d'enregistrer les candidats polonais au départ et qui, moyennant compensation financière, se livrèrent volontiers au trafic de cartes d'évacuations permettant ainsi à nombre de foyers évacués de Pologne en Ukraine de retourner en Pologne ${ }^{61}$. Ces retours impromptus cautionnèrent la propagande orchestrée par la résistance nationale ukrainienne quant à l'absurdité d'accepter le principe de l'évacuation vers l'URSS. Le message eut d'autant plus de portée qu'il provenait de véritables «volontaires », à savoir ces foyers enthousiastes de la Holmščina devenus les premières victimes des transferts de population et qui, à l'instar des Polonais arrivés d'Ukraine, dénonçaient à présent les sauvages,

57. Dès le début décembre 1944, des décès d'hommes et d'animaux liés au froid étaient constatés à l'arrivée (courrier de l'obkom de Hirov du 8 décembre 1944, Slivka, Deportaciji..., p. 372).

58. Rapport du 27 mars 1945 du NKGB d'Ukraine, sur la base de courriers interceptés (Slivka, Deportaciï..., p. 460-462).

59. CDAGOU, f. 1, op. 23, d. 1470 et d. 2605 en particulier. Les rapports sur la situation des évacués de Pologne vers l'Ukraine ont été rédigés à la suite des ordres de vérification des modalités d'application des décrets du sovnarkom d'Ukraine relatifs à l'installation des évacués (pereselency), ce qui explique leur caractère relativement tardif (à partir de juin 1945 et le plus souvent au cours de l'année 1946, voire en 1947).

60.50000 personnes seraient, selon certaines évaluations, revenues en Pologne au cours des deux années (Piotr Skrzynecki, Wysiedlenie ludności ukraińskiej z Polski w latach 1944-1946 [L'évacuation des Ukrainiens de Pologne dans les années 1944-1946], Varsovie: Myśl Pańtswowa, 1988, p. 18-19).

61. Cf. notamment CDAVOU, f. 4959, op. 1,2, d. 97, divers courriers de dénonciations par les autorités ukrainiennes. Le trafic des documents d'évacuation fut également alimenté par les attaques des administrations régionales d'enregistrement et le vol de documents vierges. 
dikie ljudi, de la grande patrie qu'ils avaient espérer enfin pouvoir retrouver. Si la faillite du plan de transplantation de la minorité ukrainienne de Pologne dans les kolkhozes d'Ukraine ne fut jamais explicitement reconnue, la réorientation, à partir du printemps 1945 des destinations des évacués, désormais conduits majoritairement vers les régions occidentales d'Ukraine d'où commençaient à partir massivement les Polonais, constituait en soi un aveu de l'échec du grand plan ukrainien ${ }^{62}$. De fait, au terme des opérations d'évacuation en juillet 1946, il apparut que seulement $30 \%$ des foyers déplacés l'avaient été vers l'Ukraine centrale et orientale, les $70 \%$ restants ayant été placés dans les régions occidentales non encore collectivisées, soit des proportions exactement inverses à celles de la planification initiale ${ }^{63}$.

\section{Conclusion sur un prélude}

Ce qui a été décrit du transfert de la minorité ukrainienne de Pologne vers l'Ukraine soviétique ne restitue que la phase initiale et « douce » de cette histoire, avant l'usage de la force qui va suivre avec l'intervention de l'armée polonaise. Or, si les pratiques répressives en tant que telles furent absentes de cette première séquence, celle-ci fut néanmoins marquée par une réelle cruauté imputable, en particulier, à la très grande carence de moyens qu'exigeait le projet pour protéger les foyers de volontaires, les transporter et les accueillir: chacune de ces étapes contint sa part de heurts et de malheurs, depuis l'agression des convois par les « bandes », le calvaire et les privations du voyage jusqu'à l'arrivée amère dans les terres de la grande misère. La politique de séduction déployée par les autorités ukrainiennes ne promettait certes pas l'abondance « immédiate » mais elle prétendait du moins pouvoir assurer la sécurité là où, en fait, les foyers évacués ne trouvèrent pas toujours de toit... Le projet de transfert économique élaboré à Kiev pour redynamiser les kolkhozes fut l'objectif assigné du plan d'évacuation qui ne connut qu'un succès très éphémère et questionnable étant donné l'absence de toute politique d'accueil. Le peu d'attention que lui ont jusqu'à présent accordé les historiens s'explique peut-être par le caractère modeste, voire dérisoire d'une telle entreprise, qui soulignait néanmoins l'état d'indigence extrême dans lequel se trouvaient les exploitations d'Ukraine. Tout apport d'hommes, d'outils et plus encore d'animaux pour relever les cheptels constituait, dans ce contexte, un véritable enjeu. Dans leurs propos «entre soi », les agents de l'État ukrainien avaient clairement explicité l'entreprise de captation des ressources attendue du plan d'évacuation. Cette dimension, passée sous silence, de l'histoire des transferts de populations, appelle un examen plus vaste des pratiques de « gestion des biens » à l'échelle de l'ensemble des territoires concernés de Pologne, mais aussi d'Ukraine. Dans une période où l'acteur étatique polonais est peu identifiable, en particulier à l'échelle locale, les pratiques de l'État ukrainien sont plus aisément

62. CDAVOU, f. 4959, op. 1.1, d. 3,1.180 sqq. sur les changements de planifications à partir du 14 mars 1945.

63. Bilan de l'évacuation des Ukrainiens de Pologne, dec. 1946, doc. cit. 
perceptibles. Elles posent différentes questions importantes pour caractériser cette « sortie » de guerre, à commencer par celle de la place tenue par les enjeux de biens dans le contentieux polono-ukrainien et l'enchaînement des violences qui a embrasé cette région. Au-delà, quelle appréciation faire de la politique ukrainienne à l'égard de son voisin et allié ? La posture de Kiev reflétait, en tous points, la conception stalinienne de la politique de «compensation» envisagée à l'égard de la Pologne : ce qu'elle perdrait à l'est, elle le retrouverait à l'ouest en se servant dans les biens des Allemands. Cette vision mécaniste des transferts, alors peu accordée au tempo du processus de libération des territoires, trouva vite ses limites dans les régions frontalières orientales avec la faillite du plan d'évacuation. L'échec y fut configuré par les conditions mêmes du déplacement, l'enchaînement des obstacles matériels aboutissant à une contre-propagande, plus efficace encore que celle de l'OUN et de l'UPA, vis-à-vis des populations concernées. Mais bien d'autres facteurs peuvent être invoqués, en particulier le «facteur géographique », s'il s'agit de nommer la diversité territorialisée des identités de la minorité ukrainienne de Pologne et la façon dont cette diversité a été perçue. Dès les premiers temps en effet, une distinction a été clairement faite entre les différents groupes installés du nord au sud de la frontière, entre les frères de sang, principalement de la Holmščina, orthodoxes et russophiles, et les étrangers «galiciens, gréco-catholiques » qui allaient effectivement constituer les principaux centres de la résistance aux « Moskales » (ainsi étaient désignés les Soviétiques dans cette région). Si cette géographie des comportements a été relevée, il convient de préciser à quel point l'affrontement à venir fut largement anticipé dans la perception différenciée et négative des Ukrainiens du sud-est de la Pologne.

Au printemps 1945, les résistances au départ et le regain d'activité des bandes nationalistes apparaissaient comme les traits dominants de la situation dans les régions frontalières. Après la tentative de susciter une émigration volontaire, devenue stagnante, le gouvernement ukrainien en vint à abandonner l'essentiel du projet de transfert économique pour se concentrer sur l'épuration politique de ces régions. L'activité anti-soviétique des résistances nationalistes encourageait celle développée de l'autre côté de la frontière. La direction ukrainienne en était venue à la conclusion que la seule possibilité de liquider les bandes était d'évacuer le plus rapidement possible la minorité ukrainienne de Pologne, considérée non seulement comme un soutien objectif à la guérilla mais aussi comme une population écran empêchant la confrontation directe avec la résistance armée. Cette analyse rejoignait celle du nouveau gouvernement polonais d'union nationale. Celui-ci, avec la fin officielle de la guerre, n'avait d'autres objectifs que d'assurer, à l'est comme à l'ouest, l'unification et la pacification du nouveau territoire polonais et prônait pour arriver à ses fins l'homogénéisation ethnique du pays. Cette convergence de vues alla de pair avec la radicalisation des résistances qui s'exprimèrent par une politique littérale de « terre brûlée ». La guerre après la guerre commençait.

Centre Marc Bloch, Berlin

CERCEC, EHESS

Catherine.Gousseff@ehess.fr 